\title{
Treatment of Cervical Spine Fractures and Subluxations without the Use of Intraoperative Fluoroscopy in Resource-Limited Settings
}

\author{
Victor-Claude Eyenga ${ }^{1}$ Ignatius N. Esene ${ }^{1,2,3} \quad$ Ernestine A. Bikono ${ }^{1} \quad$ Ngah J. Eloundou $^{1}$ \\ ${ }^{1}$ Division of Neurosurgery, Department of Surgery, Yaoundé \\ General Hospital, Yaounde, Cameroon \\ ${ }^{2}$ Neurosurgery Division, Faculty of Health Sciences, University of

\begin{abstract}
Address for correspondence Victor-Claude Eyenga, MD, PhD, MPH, Division of Neurosurgery, Department of Surgery, Yaoundé General Hospital, Yaounde, Cameroon (e-mail: dreyenga@yahoo.fr).
\end{abstract} Bamenda, Cameroon

${ }^{3}$ Neurosurgery Unit, Garoua Regional Hospital, Cameroon

J Neurosci Rural Pract 2020;11:160-163

\begin{abstract}
Keywords

- cervical spine

- fluoroscopy

- resource-limited settings

- subaxial spine

- subluxation

Background Surgical management of subaxial cervical spine injuries remains challenging. Although intraoperative fluoroscopy is usually used for intraoperative spinal level localization (SLL), it is unavailable in most developing countries. The surgeon therefore has to rely on anatomic landmarks. In our setting, in the absence of intraoperative fluoroscopy, we used the carotid tubercle for SLL. Herein we evaluate the accuracy and reliability of the carotid tubercle as a landmark during surgery for traumatic cervical spine injury.

Methods This was a retrospective cohort study on 34 patients undergoing anterior cervical surgery for subaxial cervical spine fractures and/or subluxation between January 2005 and February 2011. From their medical records, the patients' sociodemographic, clinical, radiological, and operative data were retrieved and analyzed.

Results Thirty-four patients were included in the study. The mean age was 36.2 years. Thirty patients were males. The mean duration between the trauma and surgical intervention was 9.6 days. Six patients were completely tetraplegic. Fourteen patients had fractures and 20 patients had subluxation. The carotid tubercle was palpable in all the 34 cases. Twenty-two (68.8\%) patients had partial or complete neurologic recovery. Complete anatomic reduction was achieved in 30 cases. One case of slight malalignment of the plate was observed. No case of significant deviation nor penetration of the screw into the vertebral canal was found. One patient died.

Conclusions Carotid tubercle, a palpable intrinsic marker, is an attractive anatomic landmark for SLL during surgeries for traumatic spine injuries in resource-limited settings.
\end{abstract}

\section{Introduction}

Cervical spine injuries occur in $~ 3 \%$ of all trauma patients. Injuries of the subaxial spine account for $\sim 65 \%$ of fractures and more than $75 \%$ of all dislocations. ${ }^{1}$ The goals, indications, approaches, and techniques for the treatment of cervical injuries are well established. ${ }^{2}$ However, therapeutic tools used during surgery depend on their availability.
Anterior approaches are commonly performed for traumatic cervical spine injuries. Although external landmarks can be used to estimate a general orientation along the cervical spine, fluoroscopy is usually used for intraoperative spinal level localization (SLL) ${ }^{3}$ to avoid surgical treatment of the wrong spinal level. In most developing countries and especially in rural settings, the latter is usually unavailable. The surgeon, therefore, has to rely on anatomic landmarks for 
SLL especially for patients requiring emergency surgery. The carotid (Chassaignac's) tubercle-the anterior projection of the transverse process of the sixth cervical vertebra, is a reliable anatomic landmark for the extrinsic and intrinsic SLL.-5 In our setting, in the absence of intraoperative radiographic localization equipment, we used the carotid tubercle for SLL since its position is constant and not altered with patient's position. To the best of our knowledge, this is the first study in sub-Sahara Africa evaluating the accuracy and reliability of the carotid tubercle as a landmark during surgery for traumatic cervical spine injury.

\section{Methods}

\section{Study Design}

This was a retrospective descriptive cohort study on 34 patients undergoing anterior cervical surgery for subaxial cervical spine fractures and/or subluxation at the Yaoundé General Hospital and Verjosel Clinic between January 2005 and February 2011. From their medical records, the patients' sociodemographic, clinical, radiological, and operative data were retrieved and analyzed. The Institutional (Hospital) Ethics Committee approved the study protocol. Patients who were operated for subaxial traumatic cervical spine fracture or subluxation with the use of bone graft or plates were included in the study. Patients who presented with coma and those who needed respiratory assistance were excluded. The patients' neurologic status before and after surgery was evaluated using the Frankel grading system ${ }^{6}$ from A to E. Clinical assessment was done preoperatively and at 1 month postoperatively. All included patients had a preoperative cervical spine X-ray to screen for cervical spine lesions and a computer topographic scan to confirm the lesion and a standard postoperative X-ray ( - Fig. 1).

\section{Surgical Technique}

An anterior approach was performed in all the cases. With the patient in the supine position, a standard-type pillow was placed under the shoulders to extend the neck. After sterilization and draping, a vertical skin incision, anterior to the sterno-cleido-mastoid muscle and of variable length was done. In the absence of intraoperative fluoroscopy, the carotid tubercle palpable on the anterior transverse process was used as the anatomic landmark for spinal leveling each time that the site of the injury was not spontaneously recognized by direct visualization of the contusion, hematoma, or shift in vertebral alignment. Blunt atraumatic finger dissection was done to expose the deep cervical fascia. Using the carotid tubercle, we could easily identify the level.

\section{Follow-Up}

Patient follow-up was clinical and radiological (standard cervical X-ray) in the immediate postoperative period, and at 1 month.

\section{Results}

Thirty-four patients were included in the study. The mean age \pm standard deviation [SD] was $36.2 \pm 0.4$ years (min. 18, max. 60 years). Thirty patients were males and 4 patients were females.

Concerning the preoperative management, 10 patients were placed on controlled spinal traction using Gardner-Wells' tongs; 24 patients had rigid cervical collars. The mean

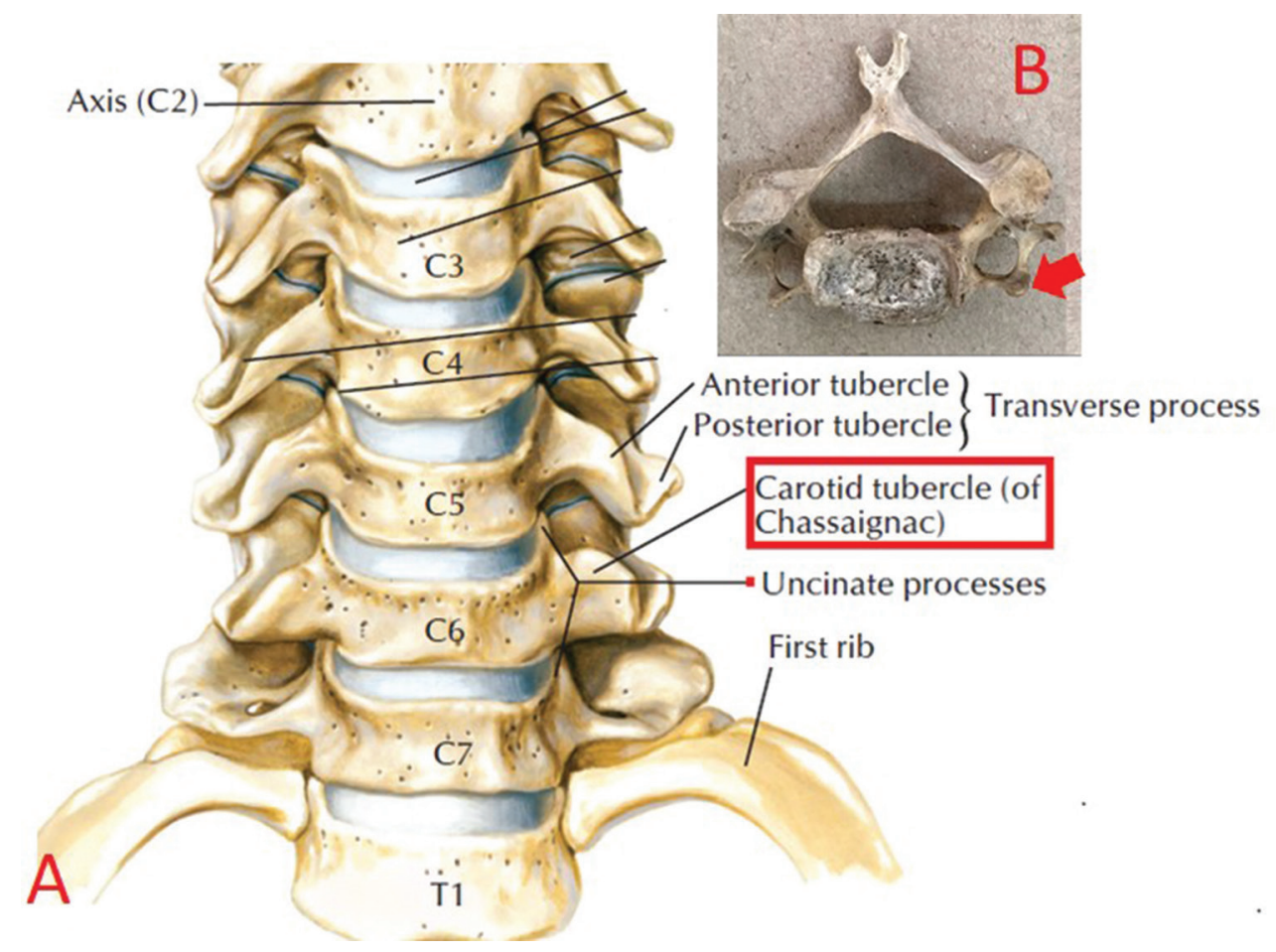

Fig. 1 Location of the carotid (Chassaignac's) tubercle. (A) The anterior projection of the transverse process of the sixth cervical vertebra (B). ${ }^{13}$ 
duration $( \pm \mathrm{SD})$ between the trauma and surgical intervention was $9.6 \pm 0.5$ days ( $\min .3$, max. 21 days).

Clinically, 6 patients were completely tetraplegic and 28 patients were with incomplete neurologic deficit.

Radiologically, fourteen patients had fractures and 20 patients had subluxation.

Intraoperatively, the carotid tubercle was palpable in all the 34 cases, all on the right side.

With regard to the clinical outcome, 22 (68.8\%) patients had partial or complete neurologic recovery and 12 (31.2\%) patients had a stationary course ( $\mathbf{- T a b l e ~} \mathbf{1}$ ).

Radiologically, complete anatomic reduction was achieved in 30 cases (-Fig. 2). Subluxation was partially reduced without vertebral canal stenosis in four cases.

Complications: We had one case of slight malalignment of the plate. No case of significant deviation nor penetration of the screw into the vertebral canal was found. We had one case of mortality on day 6 postoperation in one of the tetraplegic patients.

Table 1 Neurologic outcome (Frankel scale)

\begin{tabular}{|l|l|l|l|l|l|l|}
\hline \multicolumn{2}{|c|}{ Frankel scale } & \multicolumn{5}{|c|}{ Postoperative score } \\
\cline { 2 - 7 } \multicolumn{2}{|c|}{} & A & B & C & D & E \\
\hline \multirow{2}{*}{$\begin{array}{l}\text { Preop- } \\
\text { erative } \\
\text { score }\end{array}$} & A & 6 & 0 & 0 & 0 & 0 \\
\cline { 2 - 7 } & B & 0 & 2 & 0 & 0 & 0 \\
\cline { 2 - 7 } & C & 0 & 0 & 4 & 8 & 0 \\
\cline { 2 - 7 } & D & 0 & 0 & 0 & 0 & 8 \\
\cline { 2 - 7 } & E & 0 & 0 & 0 & 0 & $6^{\text {a }}$ \\
\hline
\end{tabular}

aFour patients had radicular pain only without myelopathy.

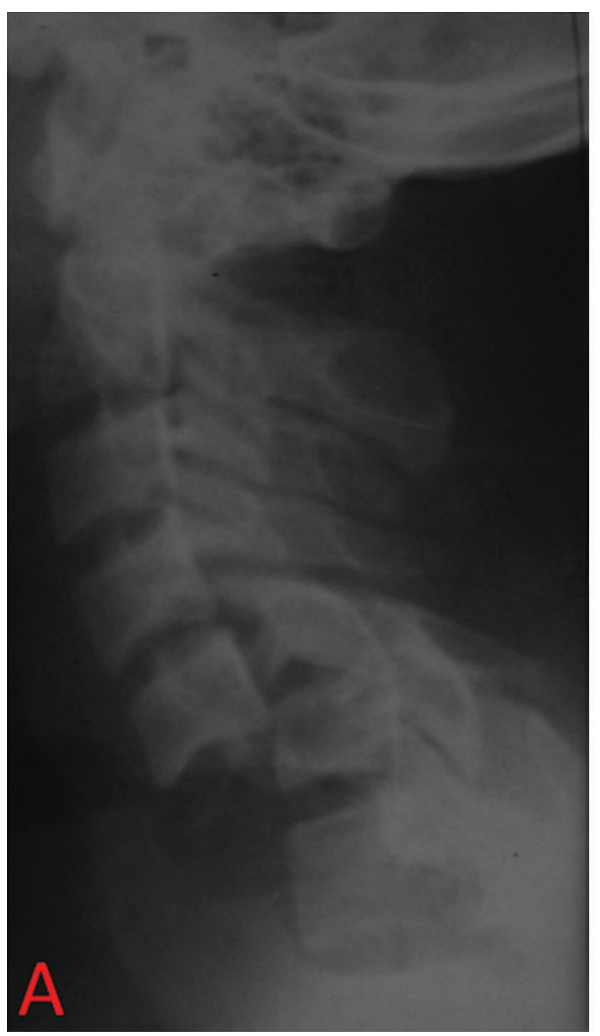

Fig. 2 C5-C6 luxation: (A) Preoperative and (B) postoperative.

\section{Discussion}

Vertebral levels of key landmarks in the neck are well documented in anatomy texts. ${ }^{5}$ Surface or extrinsic landmarks in the neck are important for planning of skin incision and orientations to the cervical vertebrae during surgery. ${ }^{7,8}$ However, these landmarks are of questionable reliability and accuracy. In a recent study, the angle of mandible, corresponding to $\mathrm{C} 2$ and $\mathrm{C} 2$ to $\mathrm{C} 3$ disc space, was the most accurate landmark for identifying the cervical levels. ${ }^{9,10}$ The hyoid bone ( $\mathrm{C} 3$ body), thyroid (C4-C5 disc), and cricoid cartilage ( $\mathrm{C} 6$ body) were not reliable in the prediction of cervical levels. ${ }^{9}$ Also, in routine clinical practice, patients are positioned with a slight neck extension to achieve cervical lordosis resulting in changes in the locations of extrinsic anatomical landmarks. ${ }^{11}$ The latter makes the use of external anatomic landmarks limited.

The Chassaignac's tubercle or carotid tubercle is an anatomic marker for SLL. As an extrinsic landmark, the Chassaignac's tubercle is characteristically palpable in the groove between the trachea and the sternocleidomastoid muscle at the $\mathrm{C} 6$ level and has been approximated to be $1.5 \mathrm{~cm}$ lateral to the midline.

As an intrinsic landmark, the carotid tubercle is the anterior prominence of the transverse process of the sixth cervical vertebra and lies lateral to and at a slightly higher level than the posterior tubercle. It separates the carotid artery from the vertebral artery as an intermediate landmark. As the carotid tubercle is intrinsic, it has the benefit of consistency in incision of the skin site that is independent from the position and extension of the cervical spine. ${ }^{12}$

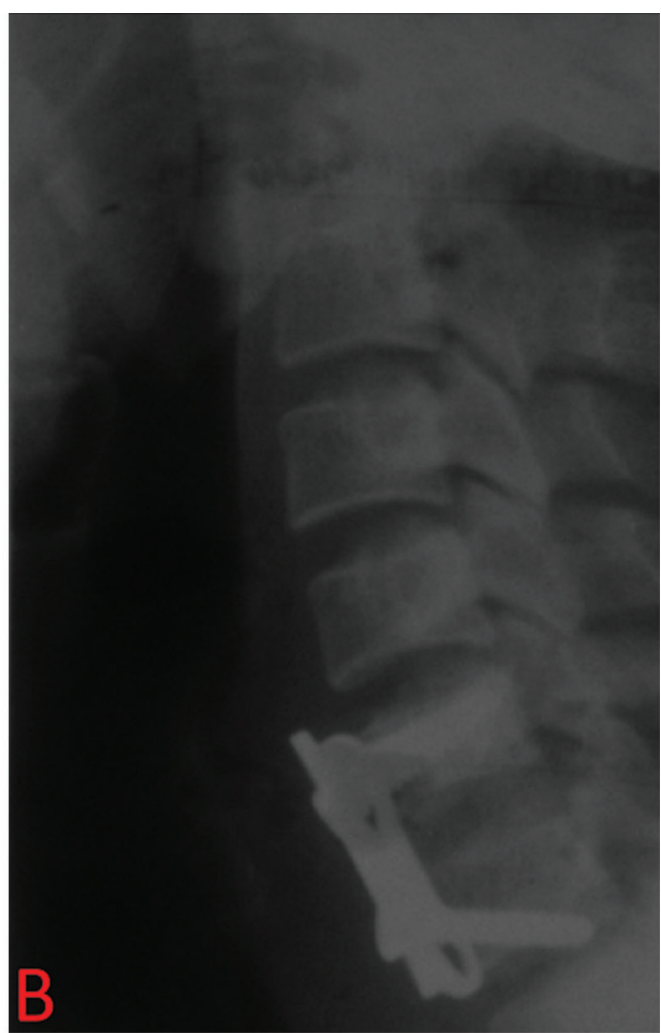


In our sample, the tubercle was identified in all the patients. Anatomic variabilities of the tubercle have been reported. In one study, the tubercles were present bilaterally $63.3 \%$ and on right side only $23.3 \%$ and left side only $13.3 \% .^{13}$

Without intraoperative fluoroscopy, we were able to effectively and safely reduce subluxation, and place screws and plates without canal violation. Subluxation was partially reduced in four cases and in one case the plate was slightly malaligned. We are unaware of any studies reporting the use of the carotid tubercle for spinal leveling during subaxial cervical spine surgery.

Clinical improvement was achieved in 22 cases (68.8\%), an outcome similar to that of Isla et $\mathrm{al}^{14}(71.4 \%)$. Our good results could be explained by the low incidence of complete spinal injury (6/34) notorious for their poor prognosis. It is well documented that patients with incomplete cord injuries recover with a better prognosis than those with complete lesions.

\section{Conclusions}

Carotid (Chassaignac's) tubercle, a palpable intrinsic marker, is an anatomic landmark for SLL during surgeries for traumatic spine injuries in resource-limited settings. Extrinsic identification of carotid (Chassaignac's) tubercle could be difficult in obese patients with short and wide necks. Although Chassaignac's tubercle can easily be palpated in the majority of patients, it cannot be identified in $30 \%$ of cases by any extrinsic landmarks, ${ }^{15}$ implying radiological guidance is needed in such cases. Anatomic variabilities of the tubercle have been reported making its identification difficult. ${ }^{13}$

\section{Ethical Approval}

This study was approved by Yaoundé General Hospital IRB authority.

\section{Conflict of Interest}

None declared.

\section{References}

1 Anderson PA, Moore TA, Davis KW, et al; Spinal Trauma Study Group. Cervical spine injury severity score. Assessment of reliability. J Bone Joint Surg Am 2007;89(5):1057-1065

2 Copley P, Tilliridou V, Jamjoom A. Traumatic cervical spine fractures in the adult. Br J Hosp Med (Lond) 2016;77(9):530-535
3 Jha DK, Thakur A, Jain M, et al. Intrinsic vertebral markers for spinal level localization in anterior cervical spine surgery: a preliminary report. Asian Spine J 2016;10(6):1033-1041

4 Lee JH, Lee JH, Lee HS, Lee DY, Lee DO. The efficacy of carotid tubercle as an anatomical landmark for identification of cervical spinal level in the anterior cervical surgery: comparison with preoperative C-arm fluoroscopy. Clin Orthop Surg 2013;5(2):129-133

5 Mirjalili SA, McFadden SL, Buckenham T, Stringer MD. Vertebral levels of key landmarks in the neck. Clin Anat 2012;25(7):851-857

6 Frankel HL, Hancock DO, Hyslop G, et al. The value of postural reduction in the initial management of closed injuries of the spine with paraplegia and tetraplegia. I. Paraplegia 1969;7(3):179-192

7 Shen XH, Xue HD, Chen Y, et al. A reassessment of cervical surface anatomy via CT scan in an adult population. Clin Anat 2017;30(3):330-335

8 Badshah M, Soames R, Ibrahim M, Khan MJ, Khan A. Surface anatomy of major anatomical landmarks of the neck in an adult population: a CT evaluation of vertebral level. Clin Anat 2017;30(6):781-787

9 Liu JM, Du LX, Xiong X, et al. Radiographic evaluation of the reliability of neck anatomic structures as anterior cervical surgical landmarks. World Neurosurg 2017;103:133-137

10 Auerbach JD, Weidner Z, Pill SG, Mehta S, Chin KR. The mandibular angle as a landmark for identification of cervical spinal level. Spine 2009;34(10):1006-1011

11 Siribumrungwong K, Sinchai C, Tangtrakulwanich B, Chaiyamongkol W. Reliability and accuracy of palpable anterior neck landmarks for the identification of cervical spinal levels. Asian Spine J 2018;12(1):80-84

12 Lee K, Lee KM, Park MS, Lee B, Kwon DG, Chung CY. Measurements of surgeons' exposure to ionizing radiation dose during intraoperative use of C-arm fluoroscopy. Spine 2012;37(14):1240-1244

13 Umakanth K, Babu KY, Mohanraj KG. Morphological and morphometrical analysis of Chassaignac's tubercle in dry human cervical vertebrae. Drug Intervent Today 2018;10(10):1943-1945

14 Isla A, Pérez-López C, Moraleda S, Martínez-Moreno M, Alvarez F, Budke M, García-Reneses J. Anterior approach in low cervical fractures prodited by hyperflexion or tear drop. Neurocirugia (Astur). 2004;15(4):360-5

15 Cha YD, Lee SK, Kim TJ, Han TH. The neck crease as a landmark of Chassaignac's tubercle in stellate ganglion block: anatomical and radiological evaluation. Acta Anaesthesiol Scand 2002;46(1):100-102 